\title{
Dimensiones de la demanda turística española: caracterización de las comunidades autónomas según la motivación de los viajeros españoles (2016)
}

\author{
Maria Jesús González González* María Eva Vallejo Pascual** \\ Universidad de Léon (España)
}

\begin{abstract}
Resumen: El objetivo de este trabajo es la identificación de las dimensiones que motivan la demanda turística española, según la comunidad autónoma de destino, a partir de los viajes realizados por los viajeros españoles recogidos por la Encuesta de Turismo de Residentes (ETR) para el año 2016, que elabora el Instituto Nacional de Estadística. La naturaleza de los datos requiere el empleo de una metodología estadística apropiada: la técnica factorial denominada Análisis de Correspondencias Simples. Como resultado del análisis se han identificado dimensiones de demanda relacionadas con el ocio: búsqueda de sol y playa; turismo de naturaleza y/o deportivo, y turismo cultural. Y también dimensiones de motivación profesional y personal: motivos profesiones y visita a familiares y amigos. Estas dimensiones permiten distinguir y caracterizar las comunidades autónomas españolas según la demanda turística nacional, analizando también su posicionamiento competitivo relativo en relación con la diversidad y estructuración de su oferta turística. Los resultados obtenidos son punto de partida para otras investigaciones de años posteriores, dado que año analizado es el primero con datos completos según la metodología ETR.
\end{abstract}

Palabras Clave: Demanda turística; Comunidades autónomas españolas; Caracterización; motivación de los viajeros; Análisis de correspondencias simples.

Dimensions of the Spanish tourist demand: characterization of the autonomous communities according to the motivation of Spanish travelers (2016)

Abstract: The objective of this study is to identify the dimensions that motivate Spanish tourist demand by autonomous community of destination, from the trips as made by Spanish travellers recorded by the Resident Tourism Survey (ETR) for the year 2016, as prepared by the National Statistics Institute. The nature of the data requires the use of appropriate statistical methodology: the factorial technique called Simple Correspondence Analysis. After this analysis, the dimensions of the demand related to leisure were identified as pursuit of sun and sand; nature and / or sports tourism, and cultural tourism together with professional reasons and personal dimensions of visits to family and friends. These dimensions allow us to characterise each Spanish autonomous community according to national tourism demand, and to analyse their relative competitive position in relation to the diversity and structure of their tourism offer. The results obtained are a starting point for other research in subsequent years, given that the year analysed is the first with complete RTE data.

Keywords: Tourist demand; Spanish autonomous communities; Characterisation; Motivation of the traveller; Simple correspondence analysis.

\section{Introducción}

La calidad de un destino turístico engloba a las empresas turísticas, los prestadores locales, los comercios, la actitud de los residentes, la infraestructura, los agentes públicos, el medio natural y el medio cultural. Todos los actores sociales deben estar involucrados en la búsqueda de la sostenibilidad, por lo que deben definirse modelos de desarrollo turístico desde una perspectiva integral (Anselin,

\footnotetext{
* Universidad de Léon (España); E-mail: mjgong@unileon.es; https://www.researchgate.net/profile/Maria_JesusG

* Universidad de Léon (España); E-mail: mevalp@unileon.es
} 
1995), desde una mirada holística, que incluya elementos y factores tan disímiles como el territorio, los alojamientos, la infraestructura y su velocidad de renovación, la densidad de población y de edificación, el clima, el valor paisajístico (Yazigi, 2002, 2003), la estacionalidad, etc. Esto debe hacerse con el consenso de los organismos públicos a los distintos niveles jurisdiccionales, desde el nacional al local, y sumando a los prestadores privados, a los actores sociales locales y aún a los propios turistas en la medida de lo posible (López Sánchez, 2012; Antón y González, 2011).

La proliferación de hospedajes ha propiciado que el turismo pase a ser una actividad interesante para la economía regional, ya que el sector turístico tiene una contribución notable al Producto Interior Bruto (PIB). Esto se observa en buena parte de las comunidades autónomas de España (Sánchez Martín et al, 2018). La infraestructura turística española es extraordinaria y constituye su principal patrimonio. Ésta cuenta con numerosos hoteles que ofrecen plazas de alojamiento que se completan con las plazas de camping, apartamentos, etc. Su distribución geográfica es desigual por comunidades autónomas y comarcas; se da una especial concentración en los espacios insulares y litorales, los más demandados por el modelo turístico. La demanda de los españoles ha cambiado en las últimas décadas hacia un turismo más cultural (motivado por el deseo de ampliar conocimientos, asistir a actos culturales, monumentos, exposiciones, museos), ecológico (visita de espacios culturales y naturales poco esperados por la acción del hombre, que pueden tener fines educativos), religioso (peregrinaciones, santuarios, viajes para asistir a celebraciones religiosas), de salud (enfocada a la curación mediante las asistencias a centros especializados, como balnearios), deportivo (dirigido a la práctica de deportes, ya sean de aventura, tradicionales, de nieve, acontecimientos deportivos), rural (enfocado a alejarse de la vida urbana y acercarse a la vida rural) y en último lugar de sol y playa(centrado en las zonas costeras en época de verano) (Dredge y Jamal, 2015). Por tanto, se trata de poner de manifiesto qué comunidades autónomas se identifican en mayor medida con estas motivaciones de viaje.

La calidad territorial también es importante a la hora de elegir una comunidad u otra por los visitantes, ya que cada vez más los españoles optan, además de los destinos tradicionales, por lugres con recursos culturales y actividades relacionadas con el ocio (Buckley,2011), señalando la tendencia a convertir los territorios en escenarios turísticos como modo de desarrollo de la sociedad del ocio, señalando como interés primordial para los territorios: valorar, ponderar y conocer la capacidad de acogida de los recursos susceptibles de ser movilizados (Canoves et al, 2014); pues existe cierta insistencia en destacar los recursos patrimoniales localizados en el territorio, «de carácter único e irrepetible», tanto natural como cultural (Pilliet Capdepón, 2010:347), con el objetivo de que puedan lograr registros tan conocidos, como Patrimonio de la Humanidad, Parque Nacional, Reserva de la Biosfera, Bien de Interés Cultural, etc, es decir, necesidad por convertirlos en patrimonio o en producto turístico (Silva y Fernández, 2008: 73)

El objetivo de este trabajo es la identificación de las dimensiones que motivan la demanda turística española, según la comunidad autónoma de destino, a partir de los viajes realizados en el año 2016 por los viajeros españoles. Existen muchas propuestas metodológicas de cómo recoger, almacenar, ordenar y clasificar la información asociada a los recursos tanto naturales como culturales o de otro tipo (Viñals et al., 2011; Burkart y Medlink,1986; Cawley y Gillmor, 2008). De hecho desde la década de los años sesenta del siglo XX en que se produce el "boom turístico" se ponen de manifiesto diferentes metodologías como la de Defert (1960), siendo la de la organización de Estados Americanos (1978) la más utilizada por autores dedicados a estos temas. Posteriormente, la Organización Mundial del Turismo (1978) incorpora los inventarios como uno de los instrumentos de trabajo básicos y autores como Burkart y Medlik (1986), Gunn (1988), Backman et al. (1991), Leno (1993), Nusser y Goebel (1997), (Baloblu, y McCelary, 1999) enfatizan las bondades de su utilización en los estudios turísticos. Así como una utilización profusa de los análisis geoestadísticos (Díaz Vieira, 2002; García-Palomares et al., 2015; Fang et al., 2015).

Además, el estudio planteado también se propone responder a otras preguntas sobre las comunidades autónomas españolas como receptoras de viajeros nacionales:

- ¿Son las actividades relacionadas con el ocio las responsables de la demanda turística de estos viajeros?

- Cuando la motivación principal es la búsqueda de sol y playa, ¿a qué comunidades se dirigen? ¿a las comunidades de mayor competitividad turística en este tipo de turismo?

- En los viajes con destino actividades deportivas y/o turismo de naturaleza, ¿sería interesante plantear otras ofertas turismo?

- Los viajes con destino comunidades sin litoral, ¿responden a una motivación cultural, basada en su oferta de patrimonio artístico? 
Planteado el estudio, en primer lugar, se revisará la información seleccionada (procedente de la encuesta de turismo de residentes (ETR) para el año 2016) y se hará una breve referencia a la técnica estadística multivariante que se aplica (análisis de correspondencias simples), así como su adecuación para el análisis de dicha información. En segundo lugar, se analizan los resultados obtenidos (numéricos y gráficos), así como su interpretación. En el apartado de discusión se dará respuesta a las preguntas planteadas, además de valorar otros aspectos que seguramente permitan abrir nuevos horizontes para la investigación de la demanda turística. El hecho de que este trabajo analice el número de viajes del primer año natural completo que es recogido por la ETR aporta un punto de partida adecuado para otras investigaciones futuras.

Los motivos que definen dichas dimensiones se relacionan con el ocio en su gran mayoría: la búsqueda de sol y playa; el turismo de naturaleza y/o deportivo, y el turismo cultural. Por último, se analizarán las conclusiones obtenidas respecto a las comunidades autónomas.

\section{Metodología: información y tratamiento}

La fuente de datos considerada para el análisis es la Encuesta de Turismo de Residentes (ETR) publicada por el Instituto Nacional de Estadística (INE). Se trata de una encuesta continua cuyo objetivo principal es proporcionar estimaciones mensuales, trimestrales y anuales de los viajes realizados por la población residente en España y sus principales características (INE, 2017). Esta encuesta, disponible a partir de febrero de 2015, da continuidad a la antigua Estadística de Movimientos Turísticos de los españoles (FAMILITUR), siendo los datos del año 2016 analizados en este trabajo los que corresponden al primer año natural completo. Entre ellas, destaca el número de viajes realizados por los viajeros españoles con destino interior, que suponen la base del resto de las variables analizadas (duración media, gasto total, gasto medio por persona, etc.). Precisamente, tomando en cuenta estas dos características, los datos objeto de análisis en este trabajo presentan como unidad de medida el número total de viajes, clasificados según el motivo principal (y por tanto, excluyente) y comunidad autónoma de destino (INE, 2016:23) para el primer año completo de la encuesta ETR.

Una tabla o matriz de grandes dimensiones como la descrita, cuyos totales filas y columnas son interpretables, no puede analizarse observando cada celda individualmente ni tampoco con la aplicación de técnicas estadísticas univariantes. De hecho, son las técnicas estadísticas multivariantes las que resultan apropiadas para analizar y describir este tipo de información. Concretamente, la técnica apropiada es la técnica factorial Análisis de Correspondencias Simples (ACS) debido a la naturaleza descrita de filas y columnas, cuyo desarrollo completo y las pautas interpretativas se pueden encontrar en Geenacre (2008) y Saporta (2013). Para la adecuada aplicación de la técnica se han considerado únicamente como modalidades activas (A) aquellas filas-motivos de los que se dispone de suficiente información para las 17 comunidades autónomas (matriz resultante de 11 filas por 17 columnas). El resto de las filas (motivos) se han considerado como suplementarias (Geenacre, 2008:126), dado que resultan de agregar varias filas (S) o presentan muchos valores ausentes (s), evitando así su exclusión del análisis (cita). Aun así, ha resultado pertinente excluir la modalidad turismo por compras (E) dado que se corresponde únicamente con la Comunidad Autónoma de Madrid. Los datos analizados, las etiquetas empleadas y su papel en el análisis se recogen en el Anexo (Tabla 1anexo). Las principales pautas interpretativas del ACS se recogen en el anexo (Tabla2anexo), y su aplicación a la matriz de datos descrita permite:

- Dotar de significado a las dimensiones (o factores) que identifican los motivos principales de los viajeros españoles con destino nacional, y que, por tanto, constituyen las dimensiones de la demanda turística española.

- Caracterizar las comunidades autónomas respecto a las dimensiones anteriormente identificadas.

- Responder a las cuestiones planteadas en el estudio, mediante un análisis adecuado de la información disponible.

Para el tratamiento de la información, se ha empleado el software estadístico el SPAD 9.0. (Coheris SPAD, 2017). 


\section{Resultados}

Se presentan y describen según el orden establecido en el anexo (Tabla2anexo), comenzando por la tabla de valores propios. Tabla 1: Valores propios, porcentaje de inercia y porcentaje
acumulado de los 3 primeros factores

\begin{tabular}{|c|c|c|c|}
\hline $\begin{array}{c}\text { Factor } \\
\text { (Dimensión) }\end{array}$ & Autovalor & Porcentaje & $\begin{array}{c}\text { Porcentaje } \\
\text { acumulado }\end{array}$ \\
\hline 1 & 0,028 & 39,7 & 39,7 \\
\hline 2 & 0,019 & 25,9 & 65,7 \\
\hline $\mathbf{3}$ & $\mathbf{0 , 0 1 2}$ & $\mathbf{1 7 , 1}$ & $\mathbf{8 2 , 7}$ \\
\hline 4 & 0,004 & 5,6 & 88,4 \\
\hline
\end{tabular}

Fuente: Elaboración propia a partir de resultados obtenidos con SPAD.9.0

El primer paso en la interpretación es determinar el número apropiado de dimensiones a retener en la solución (Bendixen, 2003). Teniendo en cuenta que la matriz de datos activos es de 11 filas y 17 columnas, cualquier dimensión retenida debe contribuir más que el máximo de los dos porcentajes siguientes: sería $100 /(11$ filas -1$)=10 \% ; 100 /(17$ columnas- 1$)=6.25 \%$. Por tanto, se retienen 3 dimensiones que representa el 82,7 \% de la información inicial.

Las dimensiones retenidas se dotan de significado según las motivaciones de viaje (modalidades-fila) de mayor contribución absoluta (CA), recogidas en la tabla 2:

Tabla 2: Motivación de viaje (etiquetas según tabla 1 anexo) de mayor contribución absoluta a las dimensiones retenidas. Puntuaciones y contribuciones absolutas: verde: puntuación positiva; roja: puntuación negativa.

\begin{tabular}{|c|c|c|c|c|c|c|c|}
\hline \multirow[b]{2}{*}{$\begin{array}{l}\text { Motivación principal de viaje } \\
\text { (modalidades-fila) }\end{array}$} & \multirow[b]{2}{*}{$\begin{array}{c}\text { Peso } \\
\text { relativo }\end{array}$} & \multicolumn{3}{|c|}{ Puntuaciones } & \multicolumn{3}{|c|}{$\begin{array}{l}\text { Contribuciones } \\
\text { absolutas (CA) }\end{array}$} \\
\hline & & Dim 1 & $\operatorname{Dim} 2$ & Dim 3 & $\operatorname{Dim} 1$ & Dim 2 & Dim 3 \\
\hline Sol y playa & 18,9 & 0,301 & 0,023 & $-0,012$ & 60,2 & 0,6 & 0,2 \\
\hline Turismo de Naturaleza & 4,5 & $-0,212$ & 0,380 & 0,091 & 7,1 & 34,8 & 3,0 \\
\hline Otros_ocio & 17,9 & $-0,059$ & 0,136 & 0,010 & 2,2 & 18,0 & 0,1 \\
\hline Otros_profesionales & 3,5 & $-0,012$ & $-0,153$ & 0,392 & 0,0 & 4,4 & 43,6 \\
\hline Cent_trabajo & 3,7 & $-0,139$ & $-0,209$ & 0,172 & 2,5 & 8,7 & 9,0 \\
\hline Fam_amigos & 35,7 & $-0,094$ & $-0,063$ & $-0,103$ & 11,1 & 7,7 & 31,1 \\
\hline Tur_deport & 2,1 & $-0,077$ & 0,326 & 0,123 & 0,4 & 11,7 & 2,6 \\
\hline Tur_cultu & 5,1 & $-0,174$ & $-0,200$ & 0,086 & 5,4 & 10,9 & 3,1 \\
\hline
\end{tabular}

Fuente: Elaboración propia con SPAD.9.0. Para cada dimensión, la suma total de CA toma el valor 100.

La interpretación de la tabla anterior permite hacer las siguientes consideraciones:

- El peso relativo permite identificar las principales motivaciones de los viajes realizados en el año 2016, sin tener en cuenta la comunidad autónoma de destino: las visitas a familiares y amigos $(35,67 \%)$, la búsqueda de sol y playa $(18,88 \%)$ y otros motivos de ocio $(17,97 \%)$.

- Según las modalidades destacadas, la dimensión 1 viene determinado por la modalidad sol y playa en su extremo positivo (60,2\% del factor), la dimensión 2 por el turismo de naturaleza y otro turismo de ocio, en su extremo positivo (conjuntamente un 52,8\%); y la dimensión 3 por 
los viajes con motivos profesiones y desplazamientos al centro de trabajo en su extremo positivo (43,6\% y $9 \%$, respectivamente).

- En cualquier caso, aunque estas son las modalidades que más contribuyen a cada factor, conviene destacar otras modalidades que presentan también una CA elevada. Así, a la primera dimensión, con contribución negativa, contribuye la modalidad que recoge los viajes cuyo motivo principal es la visita de familiares y amigos. Con puntuación positiva, también contribuye el turismo deportivo en el caso de la dimensión 2, frente a la contribución en el extremo negativo del turismo cultural (10,9 \%). En el caso de la dimensión 3, la visita a familiares y amigos contribuye de manera significativa en el extremo negativo $(31,1 \%)$.

- Las modalidades correspondientes a motivos personales (otros_perso), desplazamientos por salud (Salud) y otros motivos, no presentan contribuciones elevadas con ninguna de las dimensiones (no se presentan en la tabla 2), hecho que indica que no son el motivo principal de desplazamiento entre comunidades autónomas. Si bien, el análisis de los planos factoriales permitirá añadir algún tipo de consideración sobre las mismas en función de su posicionamiento.

- Por tanto, las tres dimensiones retenidas se identifican con la búsqueda de sol y playa (dimensión 1), la motivación aventurera (turismo de naturaleza y deportivo) frente a la motivación cultural (dimensión 2) y con los viajes por motivo de negocios frente a visitas a familiares y amigos (dimensión 3).

El análisis de los mismos indicadores para las comunidades autónomas permite matizar las anteriores consideraciones:

Tabla 3: Comunidades autónomas (etiquetas según tabla 1 anexo).

Puntuaciones y contribuciones absolutas: verde: puntuación positiva; roja: puntuación negativa. Contribuciones relativas superiores a 0,5: negrita.

\begin{tabular}{|l|c|c|c|c|c|c|c|c|c|c|}
\cline { 3 - 11 } \multicolumn{2}{c|}{} & \multicolumn{3}{c|}{ Puntuaciones } & \multicolumn{3}{c|}{$\begin{array}{c}\text { Contribuciones } \\
\text { absolutas (CA) }\end{array}$} & \multicolumn{2}{c|}{$\begin{array}{c}\text { Contribuciones } \\
\text { relativas (CR) }\end{array}$} \\
\hline $\begin{array}{l}\text { Comunidades autónomas } \\
\text { (modalidades-columna) }\end{array}$ & $\begin{array}{c}\text { Peso } \\
\text { relativo }\end{array}$ & $\begin{array}{c}\text { Dim } \\
\mathbf{1}\end{array}$ & $\begin{array}{c}\text { Dim } \\
\mathbf{2}\end{array}$ & $\begin{array}{c}\text { Dim } \\
\mathbf{3}\end{array}$ & $\begin{array}{c}\text { Dim } \\
\mathbf{1}\end{array}$ & $\begin{array}{c}\text { Dim } \\
\mathbf{2}\end{array}$ & $\begin{array}{c}\text { Dim } \\
\mathbf{3}\end{array}$ & $\begin{array}{c}\text { Dim } \\
\mathbf{1}\end{array}$ & $\begin{array}{c}\text { Dim } \\
\mathbf{2}\end{array}$ & $\begin{array}{c}\text { Dim } \\
\mathbf{3}\end{array}$ \\
\hline Andalucía & 17,5 & 0,03 & $-0,09$ & $-0,05$ & 0,7 & 8 & 3 & 0,067 & $\mathbf{0 , 5 3 6}$ & 0,134 \\
\hline Aragón & 5,5 & $-0,02$ & 0,19 & 0,09 & 0,1 & 10,9 & 3,8 & 0,004 & 0,446 & 0,103 \\
\hline C. Madrid & 8,9 & 0,03 & $-0,2$ & 0,24 & 0,3 & $\mathbf{1 9 , 7}$ & 40,7 & 0,010 & 0,412 & $\mathbf{0 , 5 6 0}$ \\
\hline Canarias & 3,2 & 0,17 & 0,03 & 0,06 & 3,3 & 0,1 & 0,9 & 0,445 & 0,011 & 0,053 \\
\hline Cantabria & 2,2 & $-0,03$ & 0,16 & 0,03 & 0,1 & 3,2 & 0,2 & 0,014 & 0,409 & 0,018 \\
\hline Castilla y León & 8,5 & 0,01 & 0,11 & $-0,13$ & 0 & 5,4 & $\mathbf{1 1 , 3}$ & 0,002 & 0,271 & 0,370 \\
\hline Castilla-La Mancha & 11,7 & $-0,03$ & 0,01 & $-0,11$ & 0,3 & 0,1 & $\mathbf{1 1 , 7}$ & 0,039 & 0,008 & $\mathbf{0 , 7 2 5}$ \\
\hline Cataluña & 12,3 & $-0,12$ & 0,21 & 0,1 & 6,2 & 28,7 & $\mathbf{1 0 , 9}$ & 0,204 & $\mathbf{0 , 6 1 8}$ & 0,154 \\
\hline C. Valenciana & 9,8 & 0,32 & $-0,01$ & $-0,03$ & 35,5 & 0,1 & 0,6 & $\mathbf{0 , 8 8 9}$ & 0,001 & 0,007 \\
\hline Extremadura & 3,3 & $-0,02$ & $-0,11$ & $-0,19$ & 0 & 2 & $\mathbf{1 0 , 3}$ & 0,007 & 0,176 & $\mathbf{0 , 5 8 8}$ \\
\hline Galicia & 5,3 & $-0,31$ & $-0,18$ & $-0,09$ & $\mathbf{1 8}$ & $\mathbf{9 , 6}$ & 3 & $\mathbf{0 , 6 0 4}$ & 0,211 & 0,044 \\
\hline I. Baleares & 1,8 & 0,35 & $-0,05$ & 0,1 & 8,2 & 0,2 & 1,5 & $\mathbf{0 , 8 7 9}$ & 0,015 & 0,069 \\
\hline La Rioja & 1 & $-0,01$ & 0,1 & 0,03 & 0 & 0,5 & 0,1 & 0,005 & 0,309 & 0,038 \\
\hline Murcia & 2,2 & 0,14 & 0,03 & $-0,03$ & 1,4 & 0,1 & 0,2 & $\mathbf{0 , 5 0 1}$ & 0,017 & 0,030 \\
\hline Navarra & 1,8 & $-0,01$ & 0,02 & 0,08 & 0 & 0 & 1 & 0,006 & 0,006 & 0,179 \\
\hline P. Asturias & 2,3 & $-0,34$ & 0,14 & $-0,02$ & $\mathbf{9 , 4}$ & 2,3 & 0,1 & $\mathbf{0 , 6 8 4}$ & 0,109 & 0,003 \\
\hline País Vasco & 2,6 & $-0,43$ & $-0,26$ & 0,06 & $\mathbf{1 6 , 6}$ & $\mathbf{9 , 1}$ & 0,7 & $\mathbf{0 , 6 5 2}$ & 0,234 & 0,012 \\
\hline
\end{tabular}

Fuente: Elaboración propia con SPAD.9.0. Para cada dimensión, la suma total de CA toma el valor 100. Para cada comunidad autónoma, la suma de CR de las dimensiones se interpreta como la calidad de representación en el plano correspondiente (ver Tabla2anexo). 
- Como para las modalidades-fila, para cada modalidad-columna el peso relativo únicamente permite determinar las comunidades autónomas que reciben mayor de viajeros: Andalucía (17,5\%), Cataluña (12,3\%) y Castila-La Mancha (11,7\%). Por detrás, se sitúa la comunidad Valenciana (9,8\%), Madrid (8,9\%) y Castilla y León (8,5\%). Las demás comunidades presentan porcentajes inferiores: concretamente son La Rioja (1\%), Navarra (1,8 \%) y Cantabria (2,2 \%) las que menos viajeros reciben.

- En la dimensión 1 destaca la contribución absoluta elevada en la parte positiva de Comunidad Valenciana y de las Islas Baleares, frente a la contribución elevada en el extremo negativo de Galicia, País Vasco y el Principado de Asturias. Dado que se trata de una dimensión de sol y playa, las puntuaciones y las contribuciones absolutas de las comunidades autónomas sin litoral costero son nulas. También presentan contribución absoluta baja el archipiélago canario, Cataluña y Andalucía, a pesar de tener litoral.

En cuanto a la dimensión 2, destaca la contribución positiva de Cataluña y Aragón; por el extremo negativo, destacan las contribuciones de Madrid, País Vasco y Andalucía. En el caso de la dimensión 3, la contribución alta de Madrid por el extremo positivo es la más destacada (también Cataluña, aunque en menor medida); contribuciones altas (negativas), pero bastante inferiores, corresponden a Castilla-La Mancha, Castilla y León y Extremadura. El resto de las comunidades no presentan contribuciones destacadas en ninguna de las dimensiones retenidas (Canarias, Cantabria, Murcia, Navarra y La Rioja).

La representación simultánea de las modalidades-fila y modalidades-columna en los planos factoriales (figuras 1 y 2) permite establecer relaciones entre motivaciones de viaje y comunidades autónomas. Cada icono es proporcional a la calidad de representación de la modalidad en el plano (resultado de sumar las contribuciones relativas de la tabla 3 en las dimensiones que definen el gráfico).

Figura 1: Representación simultánea de motivaciones de viajes y comunidades autónomas en el primer planto factorial (dimensión 1 y dimensión 2)

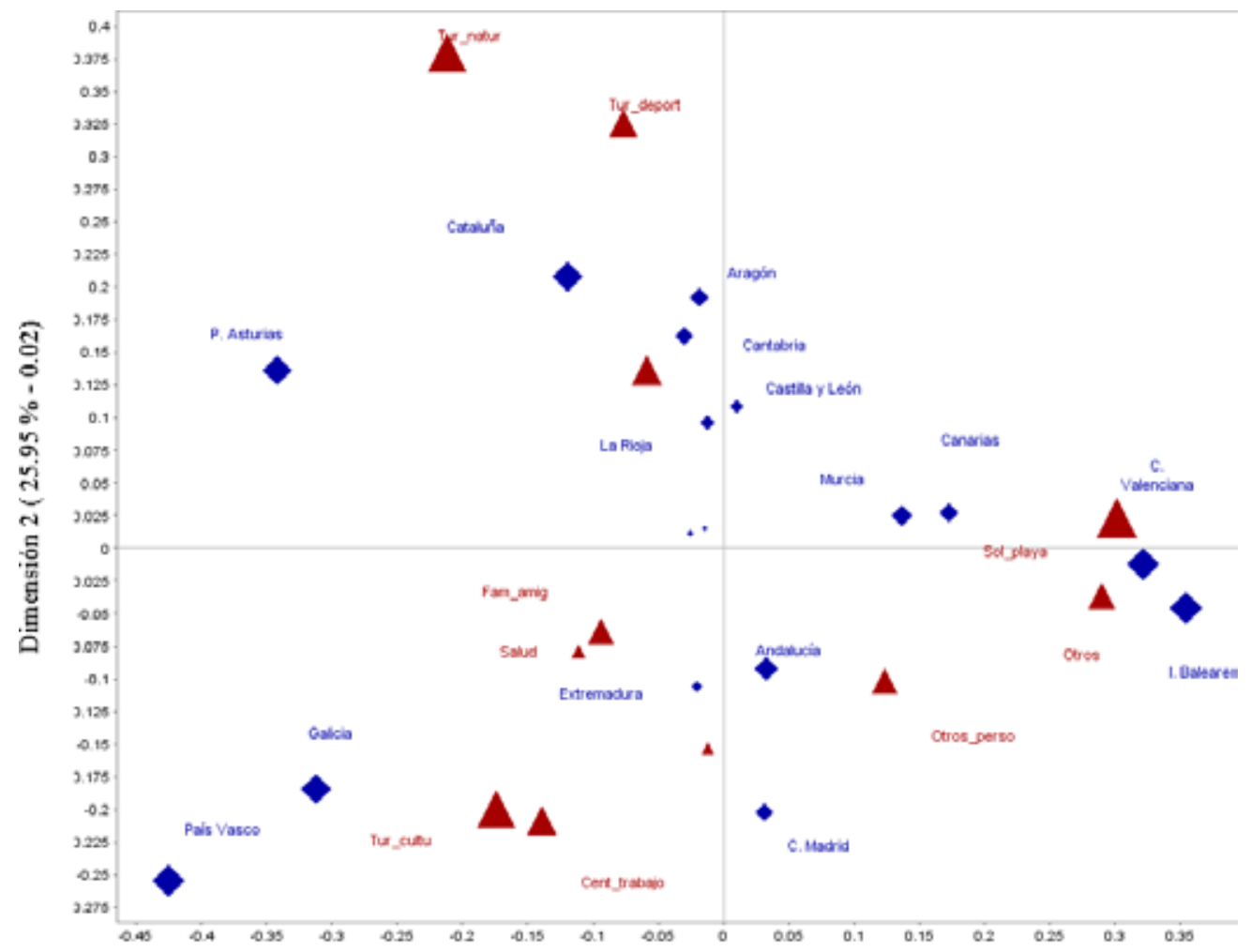

Dimensión $1(39.7 \%-0.03)$

Fuente: Elaboración propia con SPAD.9.0. Icono proporcional a su contribución relativa (CR). 
El plano representa el 65.69 \% de la información de los datos iniciales. Las posiciones destacadas (extremas) y con mayor contribución relativa (iconos grandes) en la parte positiva de la dimensión 1 son las que identifican a Baleares y a la Comunidad Valenciana como destino de los viajes cuya motivación principal es la búsqueda del sol y la playa, aunque también con viajes relacionados con otras motivaciones no específicas (distintas de las analizadas en el resto de los ítems, modalidad otros). Murcia también se identifica como destino de sol y playa, pero en menor medida que las comunidades mencionadas, teniendo en cuenta su posición menos extrema en el lado positivo (y su contribución relativa en torno al $50 \%$ de la tabla 3 ). Canarias también recibe viajeros cuya principal finalidad es el sol y la playa, pero con otro tipo de motivaciones (posición menos extrema en la dimensión y con menor calidad de representación).

Por otro lado, esta dimensión distingue claramente unas comunidades costeras de otras: Galicia, País Vasco y Asturias presentan una contribución elevada, pero en el extremo negativo de esa misma dimensión, lo que las identifica con viajes cuyo destino principal no es precisamente la búsqueda de sol y playa.

Como es obvio, las comunidades autónomas sin litoral costero presentan una posición central en esta dimensión, y su contribución relativa o calidad de representación en ese plano se debe a la dimensión 2.

- El plano permite identificar más claramente aún la asociación entre el turismo de naturaleza y el turismo deportivo (situadas en parte positiva de la dimensión 2), extremo al que contribuyen Cataluña y Aragón. Cantabria, La Rioja y Castilla y León se sitúan también en este extremo positivo, con menor calidad de representación, por lo que sólo se puede concluir que este tipo de actividad también es la motivación principal de algunos viajeros cuando visitan estas comunidades.

- La posición destacada del Principado de Asturias, en el extremo negativo de la dimensión 1 (bien representada) y positivo de la dimensión 2, la identifica con destinos de viajes que no buscan sol y playa, pero con cierta motivación por realizar actividades de turismo de naturaleza y/o deportivo.

- En cuanto a Galicia y el País Vasco, bien representadas en su posición negativa respecto a la dimensión 1, y posicionadas además en la parte negativa de la dimensión 2 , se identifican con viajeros que no buscan como actividad principal el sol y la plaza, matizándose sus posiciones en el siguiente plano (País Vasco se posicionará más cerca del turismo cultural; Galicia más cerca de la modalidad familiares y amigos).

- La posición de la modalidad centro de trabajo, bien representada en el extremo negativo de la dimensión 2 , permite afirmar que los viajes motivados por trabajo y de negocios se dirigen a comunidades como Madrid, en las que el turismo cultural también es una motivación importante.

El plano sitúa a Andalucía, bastante bien representada (la suma de las CR de las dimensiones 1 y 2 de la tabla 3 alcanza una CR de 0,6), con cierta orientación hacia el extremo negativo de la dimensión 2 , por lo tiende a recibir viajeros motivados por su atractivo cultural. Su posición más o menos central respecto a la dimensión 1, no la identifica con la búsqueda de sol y playa. 
La figura 2 representa el segundo plano factorial (dimensiones 2 y 3 ):

Figura 2: Representación simultánea de motivaciones de viajes y comunidades autónomas en el segundo planto factorial (dimensión 2 y dimensión 3)

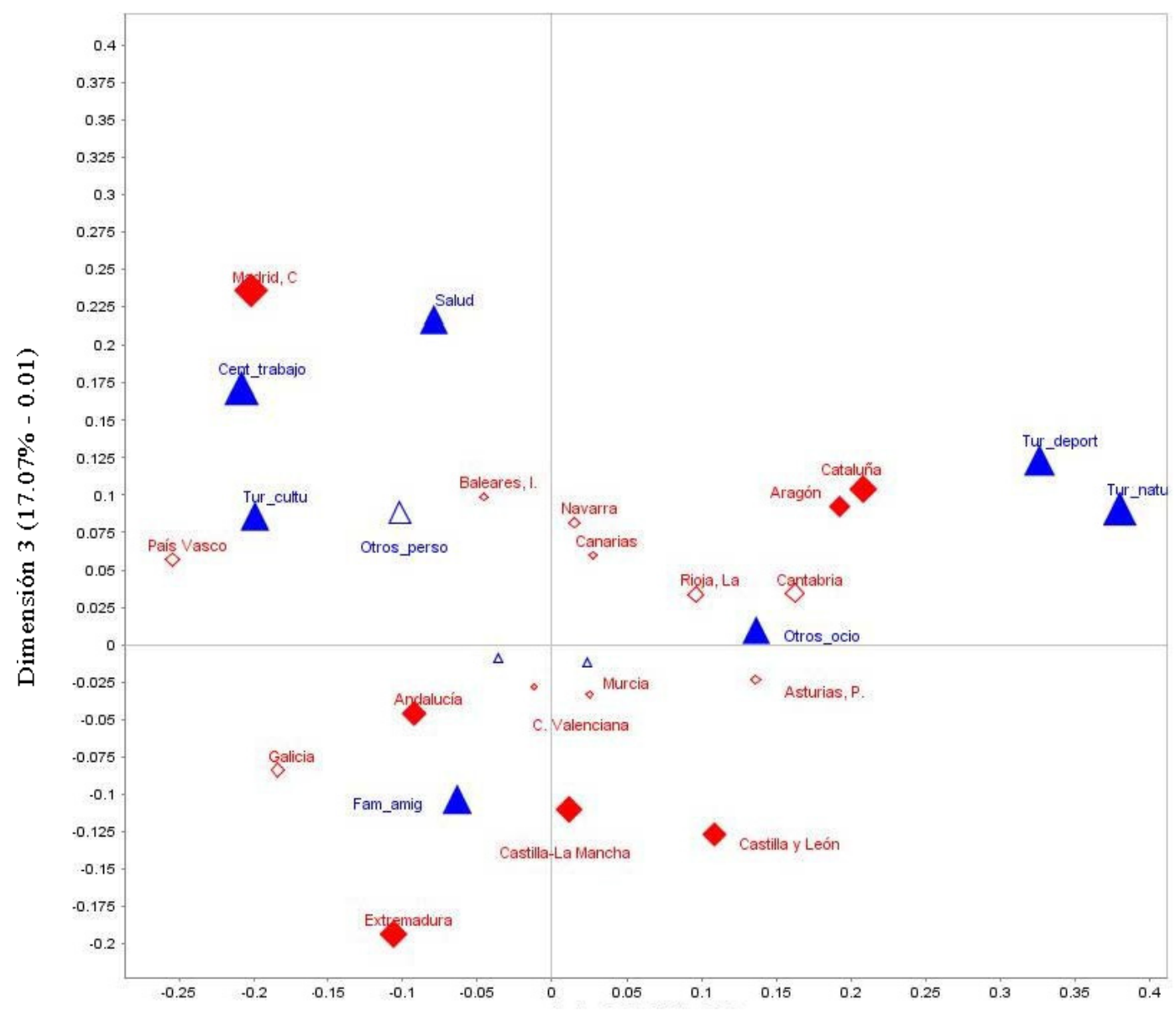

Dimensión 2(25.95\% - 0.02)

Fuente: Elaboración propia con SPAD.9.0

Este plano, que representa el 43,02 \% de la información, posiciona a la comunidad de Madrid, como la receptora de viajeros con motivos profesionales y de trabajo, de turismo cultural, así como receptora de viajes por motivo de salud. Por otro lado, el plano permite establecer aquellas comunidades en la que la visita de familiares y amigos la principal motivación: Extremadura y Castilla-La Mancha. Castilla y León está bastante bien representada en este plano (tabla 2, suma CR de 0,64 entre las dos dimensiones) y su posición en el plano la identifica con receptora de viajeros motivados por la visita de familiares y amigos, y por actividades de turismo de naturaleza y/o deportivo.

Conviene matizar un hecho ya destacado al comentar el plano anterior: el distanciamiento y pérdida de calidad de representación del País Vasco y Galicia, confirma que su cercanía se debe a que el viajero que las visita no tiene como motivo principal la búsqueda de sol y playa.

Como ya ocurría en el plano anterior, Andalucía está bastante bien representada, pero en posición central. Seguramente el motivo de esta posición es su composición: ocho provincias que de manera 
conjunta presentan todas las dimensiones identificadas: provincias con sol/playa, con turismo de naturaleza y con patrimonio cultural, por citar algunas.

Las visitas a Cantabria y a La Rioja parecen estar relacionadas con otros motivos de ocio, teniendo en cuenta su posición cercana a dicho motivo de viajes en este plano, si bien su calidad de representación es baja. Este plano factorial tampoco permite aclarar las las motivaciones de turismo de la comunidad foral de Navarra, dado que sigue estando muy mal representada.

Para finalizar la presentación de resultados, conviene añadir que de las modalidades consideradas como suplementarias en el análisis (código $s$ de la tabla1anexo: turismo gastronómico, turismo termal, otros motivos de educación, congresos y reuniones) no se presentan sus resultados numéricos ni tampoco se han presentando en los planos factoriales, dado que todas ellas ocupan posiciones centrales. De haberse incluido como modalidades activas, los resultados presentados se distorsionan dado que son modalidades con pocos efectivos (Greenacre, 2008:130).

\section{Discusión de resultados.}

Identificadas las dimensiones de la demanda turística del viajero español, se pueden añadir las siguientes consideraciones:

- El tradicional interés por el sol y/o la playa, que ha constituido la tradicional motivación del viajero extranjero (Vizcaíno Ponferrada, 2015) para visitar nuestro país, también es la motivación principal del viajero nacional para viajar a unas comunidades autónomas u otras, dado que se trata de la primera dimensión identificada.

- En segundo lugar, destaca el interés por el turismo de naturaleza y/o deportivo frente al interés por el turismo cultural. Lo que resulta destacable de esta dimensión no es su novedad, dado que la oferta de turismo de naturaleza y/o deportivo, así como el interés por conocer el Patrimonio artístico nacional, es creciente (Vizcaíno Ponferrada, 2015:84), si no su carácter opuesto en cuanto a las comunidades autónomas a las que se dirige el viajero en función de que tenga una motivación por la aventura o cultural. Este hecho plantea que la oferta de productos turísticos deba hacerse de forma diferenciada para estos dos tipos de motivaciones.

- Mientras que las dos primeras dimensiones están relacionadas con el ocio, la tercera dimensión responde a un interés profesional (negocios) y personal, teniendo en cuenta la contraposición que establece entre la motivación por trabajo (y/o negocios) y la motivación por visitar a familiares y amigos. El turismo de negocio tiene entidad propia e importante desde hace unos años y algunas ciudades concretas ya tienen cierta madurez en el mismo (Flamarich y Duro, 2011:60). Por tanto, el punto de interés de esta dimensión reside en ese tipo de viaje de motivación social/familiar que pueda combinarse con otro tipo de turismo (cultural, de naturaleza, gastronómico, etc).

Estas dimensiones permiten caracterizar las comunidades autónomas, considerando, por un lado, su posicionamiento en el análisis realizado (demanda) y, por otro, su posicionamiento competitivo relativo en relación con la diversidad y estructuración de productos turísticos ofertados (MONITUR, 2014; 2018):

- Baleares y Comunidad Valenciana son las comunidades autónomas destino principal de los españoles motivados por la búsqueda de sol y playa; sin embargo, no son las comunidades de mayor ventaja competitiva en cuanto a equipamientos playeros y playas con calidad certificada (MONITUR, 2014; MONITUR, 2018). De hecho, el País Vasco y Andalucía superan a la Comunidad Valenciana en cuanto a los equipamientos, dejando a Baleares muy por detrás (MONITUR, 2018:139). Algo parecido ocurre en cuanto a playas de calidad certificada, en las que Andalucía y Murcia superan a la Comunidad Valenciana; Baleares ocupa la quinta posición, superada también por Cantabria (MONITUR, 2018:140). En cuanto a superficie artificial del litoral, es el Principado de Asturias la de menor porcentaje (por tanto, una mayor apuesta del litoral como recurso sostenible), seguida por Baleares, Galicia, Canarias y Cantabria. En este ranking, la Comunidad Valenciana se sitúa casi al final por tener un gran porcentaje de superficie artificial en el litoral (MONITUR, 2018:142).

Por tanto, aunque las comunidades autónomas de País Vasco, Asturias y Galicia son comunidades que apuestan por poner en valor sus playas como recurso turístico, el turista español no lo percibe en la misma medida, guiándose seguramente más por su situación geográfica y meteorológica. En el caso de Andalucía, que ocupa las primeras posiciones de equipamiento pero no en cuanto a destino de sol 
y playa, la razón seguramente haya que buscarla de nuevo en la diversidad de las provincias que la componen. Quizás la comunidad autónoma de Murcia y las Islas Canarias sean las comunidades en las que sus posiciones de demanda y oferta sean más concordantes en esta dimensión de sol y playa.

En cuanto a Cataluña, sus posiciones de demanda y oferta en cuanto al turismo de sol y playa también son bastante acordes (posiciones medias en ambas). Sin embargo, Cataluña es el destino principal del viajero que demanda actividades de naturaleza y/o deportivas, hecho que se corresponde claramente con su oferta de senderos homologados, de instalaciones de recreo náuticas (MONITUR, 2018:154), de turismo de nieve (MONITUR, 2018:160) y dotación de plazas de turismo rural (MONITUR, 2018:166). En esta misma dimensión de la demanda destaca también Aragón, que está en segundo lugar, por detrás de Cataluña, en cuánto a oferta de senderos homologados y turismo de nieve. Otras comunidades autónomas, como Asturias, Cantabria y Castilla y León también tienen bastante equipamiento en cuanto a senderos homologados, instalaciones de turismo de nieve y oferta de establecimientos de turismo rural, hecho que explicaría su posicionamiento respecto a la motivación naturaleza y/o deportiva de algunos viajeros que visitan estas comunidades.

A pesar de tener ventaja competitiva en los anteriores indicadores (dotación de senderos homologados, instalaciones náuticas, turismo de nieve y turismo rural) así como en el desarrollo de vías verdes (MONITUR, 2018:152), los viajeros nacionales que visitan Andalucía suelen estar más motivados por los aspectos culturales que por el turismo deportivo y/o de naturaleza, lo que justificaría la posición de Andalucía en el plano de la figura 1. Este hecho no resulta sorprendente, dado que Andalucía ocupa la primera posición en el ranking de comunidades autónomas en cuanto al número de Bienes Patrimonio de la Humanidad (MONITUR, 2018:144). También Castilla y León lidera este ranking, pero no se refleja en su posición respecto a la demanda, pudiendo ser una cuestión a tener en cuenta para promocionar su oferta turística.

Por otro lado, dentro del turismo cultural también se incluyen las visitas a los museos, siendo líder la Comunidad de Madrid (MONITUR, 2018:146), lo que justifica claramente su posición extrema en el plano de la misma figura 1. Además, esta comunidad se situaba como receptora de gran parte de los viajes de negocio (trabajo y profesionales), lo que se traduce en su posición de líder en el ranking de competitividad en cuanto al impacto económico del turismo de negocio de viajeros españoles ((MONITUR, 2018:148). En este ranking le sigue Andalucía, aunque de nuevo ocupa una posición central en ese plano.

\section{Conclusiones}

El trabajo desarrollado y la aplicación de una técnica estadística multivariante, como es el Análisis de Correspondencias Simples, ha permitido alcanzar el objetivo planteado, identificando las tres dimensiones que motivan la demanda de viajes de los españoles en el año 2016 y dando respuesta a las cuestiones que se planteaba inicialmente este estudio.

Tal y como era previsible, los motivos que definen dichas dimensiones se relacionan con el ocio en su gran mayoría: la búsqueda de sol y playa; el turismo de naturaleza y/o deportivo, y el turismo cultural. Sin embargo, son las motivaciones de negocio y la visita de familiares y amigos las que constituyen la novedad de los resultados encontrados, ya que además son el principal motivo de viaje a las comunidades autónomas más extensas del interior de la península.

Por otro lado, entre las motivaciones de ocio que definen las dos primeras dimensiones, también hay ciertas cuestiones reseñables: según el análisis, cuando el motivo principal del viaje es la búsqueda de sol y playa, pocos viajes se dirigen a las comunidades costeras del norte-oeste peninsular, a pesar de tener una dotación excepcional de recursos. Por ello, promocionar la oferta de equipamientos y playas de País Vasco, y también de Galicia, Cantabria y Principado de Asturias, podría resultar interesante para estas comunidades autónomas.

Además, la oposición entre turismo de naturaleza-deportivo frente al turismo cultural, haría replantearse una oferta turística que combinara ambos aspectos turísticos. Así, descartada que la principal motivación del viaje sea el sol y la playa en las comunidades costeras del noroeste peninsular, el análisis también pone de manifiesto diferencias entre ellas: los viajeros que visitan a Asturias están más motivados por el turismo de naturaleza; los viajes con destino País Vasco tienen en mayor medida un motivo cultural. Por otro lado, Cataluña y Aragón destacan como principal destino de viajes con motivación por el turismo de naturaleza y deportivo, que además encaja con su oferta, lo que puede interpretarse como una adecuada orientación entre oferta y demanda turística.

Como ya se ha apuntado, las comunidades más extensas del interior peninsular son visitadas por viajeros con otros motivos. Así, una gran mayoría de los viajes con destino Madrid tienen motivos de 
trabajo y negocios, aunque también sean importantes los viajes de carácter cultural y de salud que recibe esta comunidad. Por otro, la visita de familiares y amigos es el motivo principal para viajar a Castilla y León, Extremadura y Castilla-La Mancha. Por tanto, esta última consideración sobre los viajes de motivación social/familiar pueden convertirse en una oportunidad: aprovechar esta demanda de viajeros que seguramente se produce a lo largo de todo el año, aunque seguramente centrada en fines de semana y puentes festivos, pudiendo hacer una oferta combinada con otro tipo de turismo (cultural, de naturaleza, gastronómico, etc).

El estudio también sitúa a Andalucía como la comunidad que recibe una demanda de viajeros nacionales motivados por su gran oferta turística y en todas las dimensiones: sol y playa, turismo de naturaleza y/o deportivo, turismo cultural y turismo de negocios. Esta diversidad de oferta seguramente haya que buscarla en la diversidad de sus ocho provincias.

Por tanto, el estudio realizado detalla los motivos turísticos y permite matizar la localización de la oferta de alojamientos turísticos, sea cual fuere su modalidad, lo que presenta un notable interés para determinar las políticas turísticas más adecuadas a cada espacio o para facilitar la planificación eficaz y coherente de la actividad.

Como limitaciones de este estudio se puede considerar la ausencia de caracterización de la demanda turística de la Comunidad Foral de Navarra, pero también poco definida para La Rioja o Cantabria. El motivo seguramente hay que buscarlo en su menor tamaño (en cuanto al número de viajes que reciben) y en la ausencia de tamaño muestral suficiente en algunos de los motivos que recoge la ETR del año 2016. El análisis de los datos de esta encuesta de los años sucesivos permitirá matizar las conclusiones obtenidas en este trabajo. Aun así, y dado que el 2016 constituye el primero año del que se dispone de datos según la metodología ETR, los resultados de este primer análisis se pueden considerar el punto de partida de otros estudios, que se podrán completar en la medida que estén disponibles datos para todas las comunidades autónomas relativos a otros tipos de turismo: turismo termal, turismo gastronómico, turismo enológico o turismo de compras. Por otro lado, otros indicadores, tales como el tipo alojamiento o la duración del viaje, podrán considerarse en estudios posteriores con datos de posteriores.

\section{Bibliografia}

Anselin, L. 1995. Local Indicators of Spatial Association (LISA). Geographical Analysis, 27(2): 93-115. Antón, S., y González, F. 2011. Planificación territorial del turismo. Barcelona: UOC.

Archer, B., Cooper, C., y Ruhanen, L. 2012. The positive and negative impacts of tourism. En W. Theobald, Global Tourism (pp. 79-102). Nueva York: Routledge.

Backman, S.J., Muzaffer, U. y Backman, K. 1991. Regional analysis of tourism resources. Annals of Tourism Research, 18 (2): 323-328.

Baloblu, S., y McCelary, K. 1999. A model of destination image formation. Annals of tourism research, 26(4): 868-897.

Bendixen, M. 2003. A practical guide to the use of correspondence Analysis. Marketing Research. Marketing Bulletin, 14, technical note 2. Recuperado de http://marketing-bulletin.massey.ac.nz/ V14/MB_V14_T2_Bendixen.pdf.

Buckley, R. 2011. Tourism and Environment. Annual Review of Environment and Resources, 36: 397-416.

Burkart, J. y Medlink, C. 1986. Tourism, past, present, and future. Oxford: Heinemman, London.

Cánoves, G. et al. 2014. Turismo de interior: renovarse o morir. Valencia: Universidad de Valencia.

Cawley, M., y Gillmor, D. 2008. Integrated rural tourism: Concepts and Practice. Annals of Tourism Research, 35(2): 316-337.

Coheris SPAD. 2017. Coheris Analytics SPAD 9.0. Recuperado a partir de http://www.coheris.com/ produits/analytics/logiciel-data-mining/analyse-de-donnees/.

Díaz Viera, M. 2002. Geoestadística aplicada. México: UNAM.

Defert, P. 1960. Le tourisme dans l'expansión regionale. Principes et methods. Berna: Editions Gurten, AIEST, Vol. I.

Dredge, D., y Jamal, T. 2015. Progress in tourism planning and policy: A post-structural perspective on knowledge production. Tourism Management, 51, 285-297.

Fang, Y., Huang, Z., Wang, K., y Cai, B. 2015. Spatial Pattern of Chinese Tourism Development and Its Mechanism Based on Different Spatial-temporal Scales: Taking the Panel Data of China Mainland (1996-2010) for Example. Journal of Landscape Research, 7(2): 47-54. 
Flamarich, M., Duro, J.D. 2011. "Turismo de negocios(eventos) en España: análisis y perspectivas". Papers de Turisme, pp. 59-75.

García-Palomares, J., Gutiérrez, J., y Mínguez, C. 2015. Identification of tourist hot spots based on social networks: A comparative analysis of European metropolises using photo-sharing services and GIS. Applied Geography, 63: 408-417.

Gunn, C.A. 1988. Vacationscapes: Designing tourist regions. New York:Van Nostrand Reheinhold.

Greenacre, M. 2008. La práctica del análisis de correspondencias. Bilbao: Fundación BBVA.

INE. 2016. Metodología estadística: Encuesta de Turismo de Residentes (ETR). Recuperado 30 de octubre 2017 partir de http://www.ine.es/daco/daco42/etr/etr_metodologia.pdf.

INE. 2017. Resultados detallados anuales de Viajes por principales características de los viajes (Tabla 15797). Recuperado 30 de octubre 2017 a partir de http://www.ine.es/jaxiT3/Tabla.htm?t=15797\&L=0.

Leno, F. 1993. Técnicas de evaluación del potencial turístico. Serie Libros sobre Turismo, $\mathrm{n}^{\circ} 2$, Ministerio de Industria, Comercio y Turismo. Madrid: MICYT, $261 \mathrm{pp}$.

López Sanchez, Y. 2012. Estrategias para una gestión eficaz de la designación de capital europea de la cultura como reclamo para el turismo cultural. Revista de Análisis Turístico, 14: 53-67. DOI: https:// doi.org/10.1234/RAT2011n11.

MONITUR. 2014. Monitor de la competitividad turística relativa de las comunidades autónomas españolas. Ed.: EXCELTUR. Accesible on-line:

MONITUR. 2018. Monitor de la competitividad turística relativa de las comunidades autónomas españolas. Ed.: EXCELTUR. Accesible on-line:

Nusser, S.M. y Goebel, J.J. 1997. The National Resources Inventory: a long-term multi-resource monitoring programme. Environmental and Ecological Statistics, 3(4): 181-204.

Pillet Capdepón, F. (2012): el turismo de interior en la España peninsular: el patrimonio territorial como destino turístico. Boletín de la Asociación de Geógrafos Españoles, 59:345-366.

Sánchez Martín, J. M., et al. 2018. Patrones de distribución de la oferta turística mediante técnicas geoestadísticas en Extremadura (2004-2014). Boletín de la Asociación de Geógrafos Españoles, 76 : 276-302. doi: 10.21138/bage.2523

Saporta, G. 2013. Probabilités, analyse de données et statistique ( $3^{\mathrm{a}}$ ed.). Paris: Editions TECHNIP.

Silva, R. y Fernández, V. 2008. El patrimonio y el territorio como activos para el desarrollo desde la perspectiva del ocio y del turismo. Investigaciones Geográficas, 46: 69-88.

Viñals, M. J. et al. 2011. Análisis de los criterios para la valoración turística del patrimonio natural. Investigaciones turísticas, 1: 37-50

Vizcaíno Ponferrada, M.L.,2015. "Evolución del turismo en España: el turismo cultural”. International Journal of Scientific Management and Tourism, vol 1, 4, pp. 75-95.

Yázigi, E. 2002. Turismo e Paisagem. SãoPaulo: Contexto, pp. 11-28

Yázigi, E. 2003. Turismo: uma esperança condicional. São Paulo: Global. 


\begin{tabular}{|c|c|c|c|c|c|c|c|c|c|c|c|c|c|c|c|c|c|c|c|c|}
\hline ב & & 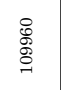 & 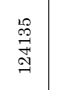 & & & & 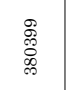 & $\begin{array}{l}\overrightarrow{\overrightarrow{0}} \\
\stackrel{0}{\circ} \\
i 0\end{array}$ & & $\begin{array}{l}\text { 总 } \\
\text { 总 }\end{array}$ & & & $\begin{array}{l}8 \\
\stackrel{8}{8} \\
:\end{array}$ & & 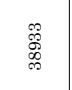 & & 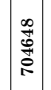 & 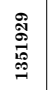 & $\underset{\infty}{\infty}$ & 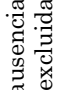 \\
\hline نि & 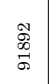 & 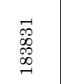 & 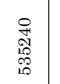 & $\begin{array}{l}\infty \\
\text { 吕 } \\
\text { స్ }\end{array}$ & 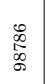 & & 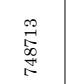 & 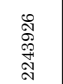 & & $\begin{array}{l}\stackrel{8}{\stackrel{8}{0}} \\
\stackrel{5}{0}\end{array}$ & & $\left|\begin{array}{|c}\mid \\
0 \\
0 \\
0 \\
0 \\
0\end{array}\right|$ & 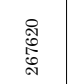 & & 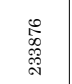 & 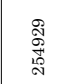 & 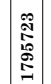 & $\begin{array}{l}\text { 器 } \\
\text { 产 }\end{array}$ & 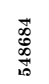 & 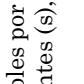 \\
\hline $\begin{array}{l}\tilde{\Xi} \\
\tilde{\Xi} \\
\tilde{Z} \\
Z\end{array}$ & & $\begin{array}{l}\mathscr{0} \\
\text { 芯 } \\
\text { 吕 }\end{array}$ & 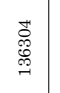 & & & & 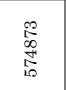 & 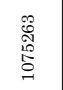 & & 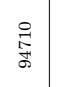 & & & 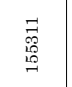 & & $\begin{array}{l}\text { o̊ } \\
\text { 今. } \\
\text { जू. }\end{array}$ & & 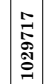 & 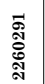 & $\begin{array}{l}\text { 藏 } \\
\text { 总 }\end{array}$ & 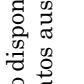 \\
\hline 莺 & $\begin{array}{l}\text { 온 } \\
\text { : }\end{array}$ & & 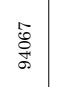 & & & & 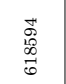 & 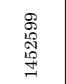 & & 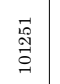 & & & $\begin{array}{l}\infty \\
\text { 岗 } \\
\stackrel{్}{్}\end{array}$ & & 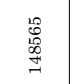 & & 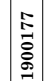 & 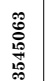 & 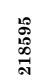 & 兽 \\
\hline ن & & 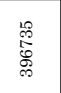 & $\begin{array}{l}\text { : } \\
\text { d. } \\
\text { d. } \\
\text { and }\end{array}$ & & 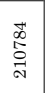 & & 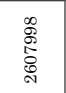 & $\begin{array}{l}\overrightarrow{\mathrm{I}} \\
\text { 骂 } \\
\text { 品 }\end{array}$ & 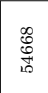 & 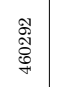 & 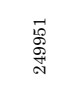 & 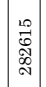 & 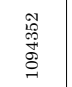 & $\begin{array}{l}\text { 氮 } \\
\text { ơ }\end{array}$ & 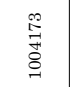 & 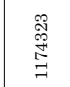 & 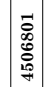 & 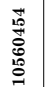 & 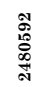 & \\
\hline 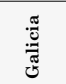 & $\begin{array}{l}\text { Oे } \\
\text { 常 }\end{array}$ & $\begin{array}{l}\text { 莕 } \\
\text { 总 } \\
\text { a }\end{array}$ & 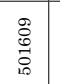 & $\begin{array}{l}\text { 菒 } \\
\text { 产 }\end{array}$ & 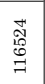 & 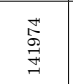 & $\begin{array}{l}\text { 是 } \\
\text { 惫 } \\
0\end{array}$ & 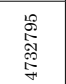 & & $\begin{array}{l}\mathscr{8} \\
\stackrel{8}{0} \\
\stackrel{\infty}{\sim}\end{array}$ & & 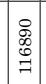 & 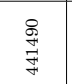 & & 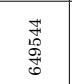 & 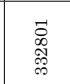 & 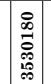 & 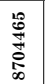 & $\begin{array}{l}\vec{E} \\
\stackrel{0}{0} \\
\Xi\end{array}$ & \\
\hline 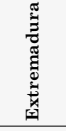 & & $\begin{array}{l}\text { o } \\
\text { ơ } \\
\text { ơ }\end{array}$ & 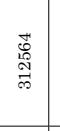 & & & & $\begin{array}{l}: \\
: 0 \\
: 0 \\
i\end{array}$ & 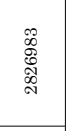 & & 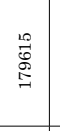 & & $\left|\begin{array}{c}: \\
\vdots \\
\vdots \\
+\end{array}\right|$ & 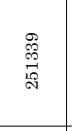 & & $\begin{array}{l}\text { 昌 } \\
\text { 尊 }\end{array}$ & $\begin{array}{l}\overrightarrow{0} \\
\stackrel{0}{\infty}\end{array}$ & 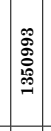 & 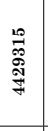 & 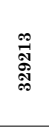 & 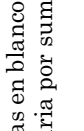 \\
\hline 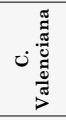 & 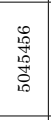 & 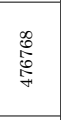 & 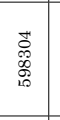 & & 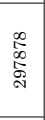 & $\begin{array}{l}\stackrel{\infty}{9} \\
\stackrel{9}{\circ} \\
\stackrel{9}{9}\end{array}$ & 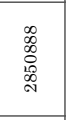 & 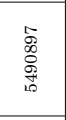 & & $\begin{array}{l}\text { 足 } \\
\stackrel{0}{0} \\
\stackrel{0}{0}\end{array}$ & & 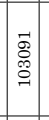 & 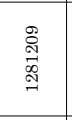 & & 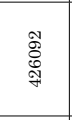 & 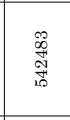 & 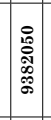 & 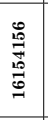 & 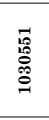 & $\begin{array}{l}0 \\
\mathbb{0} \\
0 \\
\dddot{\Xi} \\
\Im \\
-9\end{array}$ \\
\hline 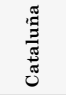 & 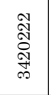 & 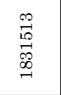 & $\begin{array}{l}\text { N. } \\
\text { 岕 } \\
\text { on }\end{array}$ & & 兽 & & 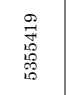 & $\begin{array}{l}\text { 울 } \\
\text { ờ } \\
\stackrel{0}{1}\end{array}$ & & 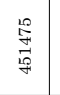 & 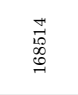 & 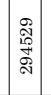 & 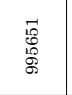 & 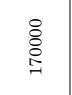 & $\begin{array}{l}0 \\
\stackrel{8}{\infty} \\
\stackrel{\infty}{\infty} \\
\infty\end{array}$ & $\begin{array}{l}\infty \\
\infty \\
\text { कू } \\
\sigma\end{array}$ & 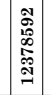 & 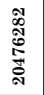 & 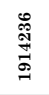 & 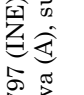 \\
\hline 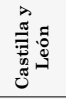 & & 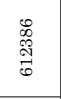 & 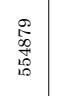 & & & & 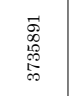 & $\begin{array}{l}\text { : } \\
\text { o. } \\
0 \\
\text { i. }\end{array}$ & & $\begin{array}{l}\text { 薄 } \\
\text { 岁 }\end{array}$ & & 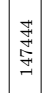 & 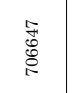 & & 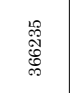 & 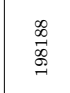 & 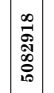 & 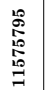 & 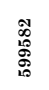 & 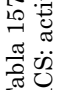 \\
\hline 离 & & 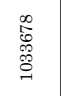 & $\begin{array}{l}\stackrel{8}{8} \\
\stackrel{8}{\Xi}\end{array}$ & 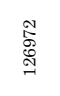 & 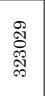 & & $\begin{array}{l}\text { 今े } \\
\text { 总 } \\
\text { 总 }\end{array}$ & 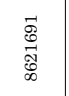 & & 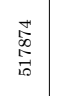 & 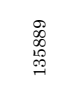 & $\mid \begin{array}{c}0 \\
0 \\
0 \\
- \\
-\end{array}$ & 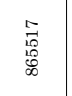 & & 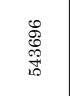 & 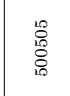 & 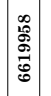 & $\begin{array}{l}\stackrel{0}{0} \\
\mathbb{E} \\
0 \\
0 \\
\end{array}$ & $\begin{array}{l}\stackrel{0}{0} \\
\stackrel{0}{0} \\
\stackrel{0}{0}\end{array}$ & \\
\hline 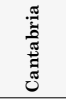 & $\begin{array}{l}\mathscr{0} \\
\text { 足 } \\
\infty \\
\infty \\
\infty\end{array}$ & 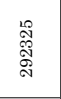 & $\begin{array}{l}\overrightarrow{0} \\
\text { o. } \\
\text { : } \\
\text { a. } \\
a\end{array}$ & & & & 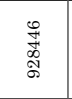 & 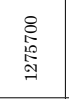 & & 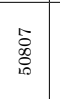 & & & $\begin{array}{l}\text { 总 } \\
\text { s. }\end{array}$ & & 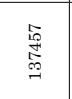 & $\begin{array}{l}\overrightarrow{\overrightarrow{0}} \\
\stackrel{0}{\stackrel{N}{N}}\end{array}$ & 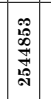 & 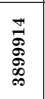 & 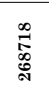 & 它 \\
\hline 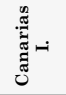 & 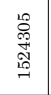 & 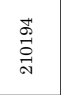 & 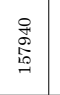 & & 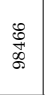 & & 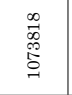 & $\begin{array}{l}\infty \\
\stackrel{\infty}{0} \\
\stackrel{0}{0} \\
\stackrel{0}{\leftrightarrow} \\
ٌ\end{array}$ & & 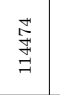 & & 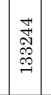 & 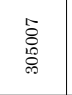 & & 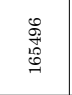 & $\begin{array}{l}\vec{H} \\
\text { 落 } \\
\text { o. }\end{array}$ & 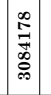 & 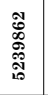 & 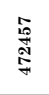 & 这 \\
\hline 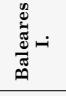 & 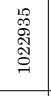 & 莟 & $\begin{array}{l}\text { 点 } \\
\text { 嵒 }\end{array}$ & & & & 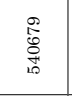 & 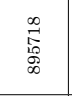 & & $\begin{array}{l}\text { 总 } \\
\text { 量 } \\
\end{array}$ & & & 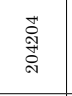 & & $\begin{array}{l}\stackrel{艹}{0} \\
\stackrel{\infty}{\exists} \\
\exists\end{array}$ & 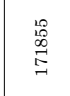 & 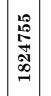 & 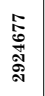 & 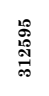 & 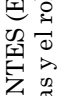 \\
\hline 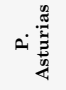 & 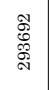 & 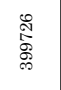 & 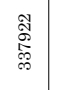 & & 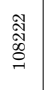 & & 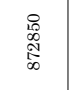 & 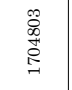 & & $\begin{array}{l}\text { 易 } \\
\text { 脱 }\end{array}$ & & & 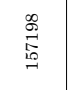 & & 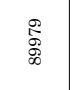 & 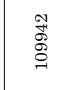 & 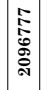 & 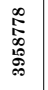 & 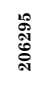 & 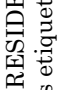 \\
\hline 总 & & $\begin{array}{l}\stackrel{8}{\circ} \\
\text { o్ } \\
\text { s. }\end{array}$ & $\begin{array}{l}\text { 间 } \\
\text { 㟧 }\end{array}$ & & 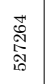 & & 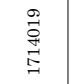 & 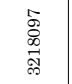 & & 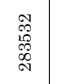 & 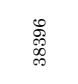 & 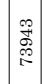 & 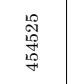 & & $\begin{array}{l}\text { 芯 } \\
\text { 品 } \\
\text { 品 }\end{array}$ & 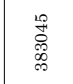 & 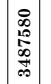 & 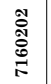 & 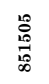 & \\
\hline 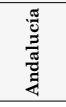 & 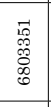 & 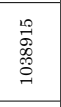 & $\begin{array}{l}\text { 吕 } \\
\text { 昜 } \\
\text { 等 }\end{array}$ & 品 & 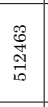 & $\begin{array}{l}\text { 获 } \\
\text { J゙ }\end{array}$ & 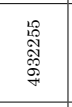 & 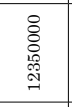 & & $\begin{array}{l}\text { 查 } \\
\text { 兽 }\end{array}$ & 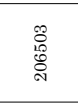 & 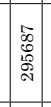 & 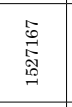 & 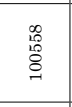 & 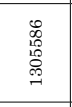 & 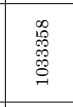 & 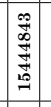 & 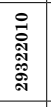 & 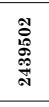 & 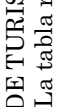 \\
\hline 웡 & $\varangle$ & 4 & 4 & $\infty$ & $\varangle$ & $\infty$ & 4 & 4 & 国 & $\varangle$ & in & $\varangle$ & 4 & $\infty$ & 4 & $\varangle$ & is & $\infty$ & $\sqrt{2}$ & \\
\hline 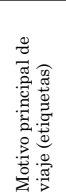 & 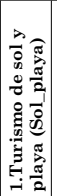 & 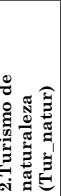 & 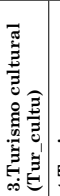 & 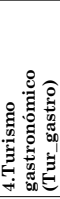 & 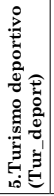 & 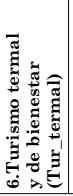 & 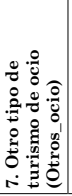 & 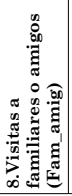 & 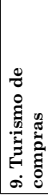 & 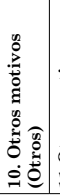 & $\begin{array}{l}0 \\
0\end{array}$ & 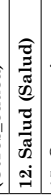 & 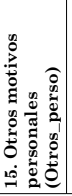 & 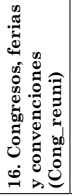 & 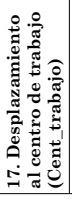 & 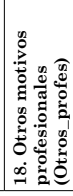 & 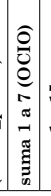 & 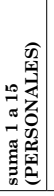 & 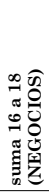 & 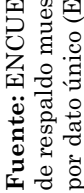 \\
\hline
\end{tabular}


Tabla 2 anexo: pautas interpretativas del Análisis de Correspondencias Simples.

\begin{tabular}{|c|c|c|}
\hline Resultados & Objetivo & Interpretación \\
\hline $\begin{array}{l}\text { Tabla de valores propios, } \\
\text { porcentaje de inercia y porcentaje } \\
\text { acumulado. }\end{array}$ & $\begin{array}{l}\text { Identificar (retener) las dimensiones } \\
\text { relevantes (denominadas también } \\
\text { factores) que representan las } \\
\text { características comunes. }\end{array}$ & $\begin{array}{l}\text { Se retienen las dimensiones que } \\
\text { explican un porcentaje acumulado } \\
\text { importante de inercia (Criterio de } \\
\text { Kaiser), que son las que han de } \\
\text { interpretarse en las fases siguientes. }\end{array}$ \\
\hline \multirow[t]{2}{*}{$\begin{array}{l}\text { Resultados por } \\
\text { modalidades- fila: puntuaciones, } \\
\text { contribuciones absolutas (CA) y } \\
\text { contribuciones relativas (CR) para } \\
\text { cada dimensión. }\end{array}$} & $\begin{array}{l}\text { Interpretar y dotar de significado a } \\
\text { cada dimensión retenida según las } \\
\text { modalidades-fila. Se analizan dos } \\
\text { indicadores: } \\
\text { 1. Contribuciones absolutas (CA) } \\
\text { de cada modalidad- fila a cada } \\
\text { dimensión. }\end{array}$ & $\begin{array}{l}\text { La suma de CA para cada dimensión es } \\
\text { vale } 100 \text {. Cada dimensión se interpreta } \\
\text { en función de aquellas modalidades- fila } \\
\text { activas con alta contribución absoluta } \\
\text { CA (superior a su peso teórico). } \\
\text { Las modalidades con pocos efectivos } \\
\text { se sitúan lejos de las dimensiones } \\
\text { comunes. Por tanto, es recomendable su } \\
\text { posicionamiento como modalidades- fila } \\
\text { suplementarias (no activas) y sólo } \\
\text { se considera su posición en el plano } \\
\text { (tienen puntuación, pero no tienen CA). }\end{array}$ \\
\hline & $\begin{array}{l}\text { 2. Puntuaciones de cada } \\
\text { modalidad- fila a cada dimensión. }\end{array}$ & $\begin{array}{l}\text { Para cada dimensión, se analiza el } \\
\text { signo de las modalidades- fila activas } \\
\text { antes destacadas: } \\
\text { (mismo signo, indica asociación } \\
\text { positiva; } \\
\text { signo contrario, indica asociación } \\
\text { negativa). }\end{array}$ \\
\hline $\begin{array}{l}\text { Resultados por } \\
\text { modalidades- columna: } \\
\text { puntuaciones, contribuciones } \\
\text { absolutas (CA) y contribuciones } \\
\text { relativas (CR) para cada } \\
\text { dimensión. }\end{array}$ & $\begin{array}{l}\text { Interpretar y dotar de significado a } \\
\text { cada dimensión retenida según las } \\
\quad \text { modalidade- columna. } \\
\text { 1. Contribuciones absolutas (CA) } \\
\text { de cada modalidad- columna en } \\
\text { cada dimensión. } \\
\text { 2. Puntuaciones de cada } \\
\text { modalidad- columna en cada } \\
\text { dimensión. }\end{array}$ & $\begin{array}{l}\text { Pautas idénticas a las relativas a } \\
\text { la interpretación de las dimensione } \\
\text { según las modalidades- fila. }\end{array}$ \\
\hline $\begin{array}{l}\text { Representación de los planos } \\
\text { factoriales: } \\
\text { gráficas de modalidades en dos } \\
\text { dimensiones, considerando las } \\
\text { dimensiones retenidas dos a dos. }\end{array}$ & \multicolumn{2}{|c|}{$\begin{array}{l}\text { Se interpretan sólo las modalidades- fila y modalidades- columna bien } \\
\text { representadas, es decir, con contribución relativa (CR) superior al } 50 \% \text { en } \\
\text { el plano (Greenacre, 2008:121). La CR es el cuadrado del coseno del ángulo que } \\
\text { forma cada modalidad con dicho plano, por lo que ese valor indica que es una } \\
\text { modalidad que forma un ángulo pequeño con el plano representado. Por tanto, } \\
\text { la suma de las CR de cada modalidad vale } 1 \text {. } \\
\text { - La proximidad entre modalidades- fila bien representadas indica que } \\
\text { están presenten en las mismas modalidades- columna. } \\
\text { - La proximidad entre modalidades- columna bien representadas indica } \\
\text { que están presenten en las mismas modalidades- fila. } \\
\text { - Las modalidades-fila (columna) cercanas al origen indican una presencia } \\
\text { similar en las modalidades-columna (fila). } \\
\text { - Las posiciones de las modalidades suplementarias se interpretan por } \\
\text { según la interpretación de cada dimensión. } \\
\text { Las modalidades con contribución relativa baja (CR inferior al 50\%) } \\
\text { no deben interpretarse dado que su posición real está lejos de las dimensiones } \\
\text { representadas (icono pequeño indica alejamiento), es decir, forman ángulos muy } \\
\text { grandes las dimensiones representadas (Greenacre, 2008:121). } \\
\text { REPRESENTACIÓN SIMULTÁNEA: La posición próxima entre modalidades- } \\
\text { fila y modalidades- columna bien representadas, se interpreta teniendo en } \\
\text { cuenta la estructura cuasi- baricéntrica del análisis: cada modalidad- columna } \\
\text { es el baricentro de las modalidades-fila con las que se asocia (y viceversa). }\end{array}$} \\
\hline
\end{tabular}

Fuente: Elaboración propia a partir de Greenacre (2008)

Recibido: 